OPEN ACCESS

Edited by:

Thomas Dick

Rutgers, The State University of New Jersey, Newark, United States

Reviewed by:

Timothy Barkham

Tan Tock Seng Hospital, Singapore Avi Peretz,

The Baruch Padeh Medical Center,

Poriya, Israel

${ }^{*}$ Correspondence:

Jeanette W. P. Teo

jeanette_teo@nuhs.edu.sg

Specialty section:

This article was submitted to Antimicrobials, Resistance and

Chemotherapy,

a section of the journa

Frontiers in Microbiology

Received: 18 July 2018 Accepted: 16 August 2018 Published: 07 September 2018

Citation: Tang YW, Cheng B, Yeoh SF, Lin RTP and Teo JWP (2018) Tedizolid Activity

Against Clinical Mycobacterium abscessus Complex Isolates - An in vitro Characterization Study.

Front. Microbiol. 9:2095. doi: 10.3389/fmicb.2018.02095

\section{Tedizolid Activity Against Clinical Mycobacterium abscessus Complex Isolates - An in vitro Characterization Study}

\author{
Ying Wei Tang ${ }^{1}$, Bernadette Cheng ${ }^{2}$, Siang Fei Yeoh ${ }^{3}$, Raymond T. P. Lin ${ }^{2,4}$ and \\ Jeanette W. P. Teo ${ }^{2 *}$ \\ ${ }^{1}$ Department of Biological Sciences, National University Singapore, Singapore, Singapore, ${ }^{2}$ Department of Laboratory \\ Medicine, National University Hospital, Singapore, Singapore, ${ }^{3}$ Pharmacy, National University Hospital, Singapore, \\ Singapore, ${ }^{4}$ National Public Health Laboratory, Ministry of Health, Singapore, Singapore
}

Mycobacterium abscessus complex consist of three rapidly growing subspecies: M. abscessus, M. massiliense, and $M$. bolletii. They are clinically important human pathogens responsible for opportunistic pulmonary and skin and soft tissue infections. Treatment of $M$. abscessus infections is difficult due to in vitro resistance to most antimicrobial agents. Tedizolid (TZD) is a next-generation oxazolidinone antimicrobial with a wide spectrum of activity even against multidrug resistant Gram-positive bacteria. In this study, the in vitro activity of TZD against the M. abscessus complex $(n=130)$ was investigated. Susceptibility testing by broth microdilution showed lower TZD minimum inhibitory concentrations (MICs) when compared to linezolid. The $\mathrm{MIC}_{50}$ and $\mathrm{MIC}_{90}$ was $1 \mathrm{mg} / \mathrm{L}$ and $4 \mathrm{mg} / \mathrm{L}$, respectively across all $M$. abscessus complex members, reflecting no difference in subspecies response to TZD. Pre-exposure of $M$. abscessus complex to subinhibitory concentrations of TZD did not trigger any inducible drug resistance. Single-drug time kill assays and bactericidal activity assays demonstrated bacteriostatic activity of TZD in all three $M$. abscessus subspecies, even at high drug concentrations of 4 to $8 x$ MIC. Combination testing of TZD with clarithromycin, doxycycline and amikacin using the checkerboard approach showed no antagonistic interactions. TZD may be an effective therapeutic antimicrobial agent for the treatment of $M$. abscessus infections.

Keywords: multidrug resistant (MDR), inducible resistance, oxazolidinone, time-kill, repurposable drugs

\section{INTRODUCTION}

Mycobacterium abscessus complex consists of three rapidly-growing mycobacteria (RGM) subspecies: M. abscessus subspecies abscessus, $M$. abscessus subspecies massiliense and M. abscessus subspecies bolletii (Lee et al., 2015). They have emerged as clinically important multi-drug resistant (MDR) human pathogens responsible for a wide spectrum of skin and soft tissue infections (SSTIs), opportunistic infections in immunocompromised patients and pulmonary infections in patients with chronic pulmonary disease or cystic fibrosis (Nessar et al., 2012). Nosocomial outbreaks of M. abscessus have been reported worldwide, highlighting its clinical significance (Nessar et al., 2012). M. abscessus complex accounts for approximately $65-80 \%$ of pulmonary infections caused by RGM (Koh et al., 2011). In Singapore, M. abscessus complex is the most prevalent RGM isolated 
in hospitals and accounts for approximately 35\% of all nontuberculous mycobacteria (NTM) infections (Tang et al., 2015).

M. abscessus pulmonary infections are infamously difficult to treat, with low cure rates ranging from 30 to $50 \%$. This is attributed to natural resistance to most antimicrobial agents (Van Ingen et al., 2012). Existing treatment regimens are combinationbased therapies usually consisting of a macrolide antibiotic such as clarithromycin (CLR), amikacin (AMK) and either cefoxitin (FOX), imipenem (IPM), or tigecycline (TGC) (Van Ingen et al., 2012). The administration of combination therapy (usually CLR and $\mathrm{AMK}$ ) is lengthy, lasting for periods of between 2 and 4 months before clinical and microbiological improvements are noticeable (Huang et al., 2010). And the lack of alternative antimicrobial options further complicates the treatment of NTM infections (Benwill and Wallace, 2014).

Tedizolid (TZD) is a next-generation oxazolidinone antibiotic approved by the Food and Drug Administration (FDA) in 2014 for the treatment of acute bacterial skin and skin structure infections (ABSSSI) caused by certain Streptococcus spp. and methicillin-resistant Staphylococcus aureus (MRSA). Phase three clinical trials demonstrated non-inferiority of TZD to the first-in-class oxazolidinone LZD for the treatment of ABSSI, with improved clinical efficacy against MRSA and slightly improved safety profile (Moran et al., 2014). Oxazolidinones are protein synthesis inhibitors (Rybak et al., 2014) whose action is primarily bacteriostatic (Rybak et al., 2014). In vitro, TZD has demonstrated activity against acid-fast bacilli such as slowgrowing Mycobacterium tuberculosis and the rapidly-growing Mycobacterium fortuitum (Kisgen et al., 2014). TZD MIC values against NTM were equivalent or 1- to 8-fold lower than those of LZD, indicating improved in vitro potency (Brown-Elliott and Wallace, 2017). Another study showed that TZD exhibited good bacteriostatic activity against M. abscessus, with MICs twoto 16-fold lower as compared to LZD (Compain et al., 2018). The combination of in vitro activity against MDR Gram-positive bacteria, an oral dosage formulation and once-daily dosing makes TZD a promising investigational antimicrobial therapeutic agent (Kisgen et al., 2014).

In this study, we explored the potential use of TZD for anti-mycobacterial therapy by characterizing the in vitro activity of TZD against 130 clinical isolates of M. abscessus complex members.

\section{MATERIALS AND METHODS}

\section{Mycobacterial Isolates and Genetic Characterization}

A total of 130 retrospective non-duplicate clinical M. abscessus complex isolates were evaluated. This collection consisted of 43 M. abscessus isolates, $82 \mathrm{M}$. massiliense isolates and five M. bolletii isolates. The subspecies of the M. abscessus complex isolates was determined by multi-locus sequencing employing the $r p o B$ and hsp65 genes (Macheras et al., 2011). CLR resistance was analyzed by full-length sequencing of the erm(41) and $r r l$ genes (Aziz et al., 2017). For erm(41), the full-length 673 bp gene sequence was examined for T/C polymorphism at the 28th nucleotide position as well as for gene deletions. erm(41) T28 sequevars have wild-type inducible CLR resistance whilst C28 sequevars are phenotypically CLR susceptible (Choi et al., 2012). For the $r r l$ gene, the nucleotides 2058-2059, associated with CLR resistance were examined.

\section{MIC Determination}

Antibiotic powders of TZD, CLR, and LZD were purchased from MedChem Express (NJ, USA). Antimicrobial susceptibility testing of TZD, CLR and LZD were performed using the microdilution method according to the Clinical \& Laboratory Standards Institute (CLSI) guidelines (CLSI, 2015). The working range for all tested antimicrobials was $0.125-64 \mathrm{mg} / \mathrm{L}$. For TZD and $\mathrm{LZD}$, the inoculated microdilution plates were incubated at $30^{\circ} \mathrm{C}$ for 3-5 days before growth was assessed by visual inspection. The MIC was determined as the concentration of antibiotic at which there was no visible growth. Staphylococcus aureus ATCC (American type culture collection) 6538 and Enterococcus faecalis ATCC 29212 were used as susceptibility testing quality control strains. The MIC for the control strains fell within the acceptable MIC range of $0.25-1 \mathrm{mg} / \mathrm{L}$ for both TZD and LZD (Woods et al., 2011; Brown-Elliott and Wallace, 2017). For TZD, there are currently no interpretative criteria for RGM. For LZD, RGM with MICs of $\leq 8$ were classified as sensitive and $\geq 32$ as resistant (Woods et al., 2011).

\section{Bactericidal/Static Activity Determination}

For the bactericidal/static activity determination assay, $M$. abscessus isolates $(n=7), M$. massiliense isolates $(n=15)$ and $M$. bolletii $(n=5)$ were tested. After three days of TZD incubation at $30^{\circ} \mathrm{C}$, the entire 96-well microtiter plate well contents corresponding to the two-fold diluted TZD concentrations $(64-0.0625 \mathrm{mg} / \mathrm{L})$ were plated and the CFU determined. The Minimum Bactericidal Concentration (MBC) of TZD against the tested isolates was defined as the lowest drug concentration required to induce $\geq 99.9 \%$ cell death as compared to the untreated control at the $0 \mathrm{~h}$ time point. For bactericidal antibiotics, the MBC is classified as $\leq 4$ times the MIC while the $\mathrm{MBC}$ is usually $>4$ times the MIC for bacteriostatic antibiotics.

\section{TZD Time Kill Assay for the M. abscessus Complex}

Time-kill assays were performed according to CLSI guidelines (CLSI, 1999) and were setup for a single isolate each of $M$. abscessus, M. massiliense and $M$. bolletii using a $10^{6} \mathrm{CFU} / \mathrm{mL}$ inoculum exponential growth phase bacterial suspension. Twofold increasing concentrations of TZD (from 0.25 to $8 \mathrm{x}$ MIC) and a drug-free growth control was used. At time intervals of 0 , $4,8,12,24,36,48,72,96$, and $120 \mathrm{~h} \mathrm{CFU}$ enumerations were made. Bactericidal activity was defined as a $\geq 3-\log _{10}$ decrease in $\mathrm{CFU} / \mathrm{mL}$ at $120 \mathrm{~h}$ when compared to the $0 \mathrm{~h}$ time point. All timekill experiments were performed in duplicate and the mean CFU counts plotted.

\section{TZD Pre-exposure Assay}

erm(41) confers inducible macrolide resistance in the $M$. abscessus complex, observable phenotypically at day 14 of 
TABLE 1 | MICs of tedizolid, clarithromycin and linezolid for 130 clinical isolates of Mycobacterium abscessus complex.

\begin{tabular}{|c|c|c|c|c|c|}
\hline $\begin{array}{l}\text { Antimicrobial } \\
\text { agent }\end{array}$ & M. abscessus complex $(n=130)$ & $\mathrm{MIC}_{50}$ & $\mathrm{MIC}_{90}$ & MIC range (mg/L) & Susceptibility (\%)* \\
\hline \multirow[t]{4}{*}{ Tedizolid } & M. abscessus (43) & 1 & 4 & $0.0625-8$ & N/A \\
\hline & M. bolletii (5) & 4 & 4 & $1-8$ & $\mathrm{~N} / \mathrm{A}$ \\
\hline & M. massiliense (82) & 1 & 4 & $0.0625-8$ & N/A \\
\hline & Total (130) & 1 & 4 & $0.0625-8$ & N/A \\
\hline \multirow[t]{4}{*}{ Linezolid } & M. abscessus (43) & 8 & $>32$ & $0.0625->32$ & 53.5 \\
\hline & M. bolletii (5) & 32 & $>32$ & $8->32$ & 20 \\
\hline & M. massiliense (82) & 8 & $>32$ & $0.5->32$ & 53.7 \\
\hline & Total $(130)$ & 8 & $>32$ & $0.0625->32$ & 52.3 \\
\hline \multirow[t]{4}{*}{ Clarithromycin* } & M. abscessus (43) & $>16$ & $>16$ & $0.0625->16$ & 20.9 \\
\hline & M. bolletii (5) & $>16$ & $>16$ & $1->16$ & 40 \\
\hline & M. massiliense (82) & 0.5 & 12 & $0.0625->16$ & 76.8 \\
\hline & Total (130) & 1 & $>16$ & $0.0625->16$ & 55.4 \\
\hline
\end{tabular}

"For $L Z D$, isolates with MICs of $\leq 8$ were classified as sensitive and $\geq 32$ were resistant (Woods et al., 2011).

For CLR, isolates with MICs of $\leq 2$ were classified as sensitive and $\geq 8$ were resistant.

incubation (Rubio et al., 2015). To examine if a similar inducible phenomenon existed for TZD, $M$. abscessus complex isolates were pre-exposed to sub-inhibitory concentrations of TZD prior to MIC determination as previously described (Aziz et al., 2017). TZD pre-exposure assays were performed for three isolates each of M. abscessus, M. massiliense and M. bolletii. Briefly, $10^{6}$ CFU/mL bacterial suspension was treated with TZD at a subinhibitory concentration of $0.25 \mathrm{mg} / \mathrm{L}$ for $M$. abscessus and $M$. massiliense isolates, and at $1 \mathrm{mg} / \mathrm{L}$ for $M$. bolletii isolates, fourfold lower than their $\mathrm{MIC}_{50}$ values. An untreated, drug-free culture was setup as a growth control. The MICs were determined at day 3 and at day 14 .

\section{Synergy Studies Using Checkerboard Titration Assay}

The in vitro interactions of TZD and CLR, TZD and DOX, as well as TZD and AMK were investigated by the checkerboard approach using the broth microdilution method as previously described (Kaushik et al., 2015). Five isolates of $M$. abscessus, four M. massiliense and five $M$. bolletii isolates were used for evaluation. The fractional inhibitory concentration index ( $\sum$ FIC) for each isolate was calculated as follows: $\sum$ FIC $=\frac{\text { MIC of antibiotic } 1 \text { in combination }}{\text { MIC of antibiotic } 1 \text { only }}+$ $\frac{\text { MIC of antibiotic } 2 \text { in combination }}{\text { MIC of antibiotic } 2 \text { only }}$. Synergy was defined as a FIC index of $\leq 0.5$, indifference by a FIC index of $>0.5$ to $\leq 4$ and antagonism when the FIC index was $>4$.

\section{RESULTS}

\section{Susceptibility of $M$. abscessus Complex Isolates to Tedizolid, Clarithromycin, and Linezolid}

For TZD, the MIC range was $0.0625-8 \mathrm{mg} / \mathrm{L}$, compared to $0.0625->32 \mathrm{mg} / \mathrm{L}$ for LZD. The $\mathrm{MIC}_{50}$ and $\mathrm{MIC}_{90}$ for TZD was 1 and $4 \mathrm{mg} / \mathrm{L}$, consistent across all three subspecies suggesting that the 3 subspecies were similarly responsive to TZD. In general, the TZD MICs were 2- to 16-fold lower than those of LZD. Due to the lack of interpretive criteria for TZD for RGM, susceptibility rates were not assigned (Table 1). For LZD, 52.3\% of all isolates were susceptible (MIC $\leq 8 \mathrm{mg} / \mathrm{L}) ; 53.5 \%$ of $M$. abscessus, $20 \%$ of $M$. bolletii and $53.7 \%$ of $M$. massiliense (Table 1).

For CLR, the $\mathrm{MIC}_{50}$ and $\mathrm{MIC}_{90}$ were both $>16 \mathrm{mg} / \mathrm{L}$ for M. abscessus and M. bolletii, as compared to 1 and 12 $\mathrm{mg} / \mathrm{L}$ for M. massiliense. According to CLSI interpretive criteria for susceptibility (CLSI, 2017), 55.4\% (72/130) of all isolates were susceptible to CLR (MIC $<2 \mathrm{mg} / \mathrm{L}$ ). M. massiliense isolates showed susceptibility rates of $76.8 \%$. This is consistent with the observation that $M$. massiliense usually possesses a truncated non-functional erm(41) (Chew et al., 2017). In contrast, $M$. abscessus (20.9\% susceptible) and $M$. bolletii isolates ( $0 \%$ susceptible) showed higher rates of resistance to CLR. The susceptible M. abscessus isolates were erm(41) C28 sequevar.

\section{TZD Does Not Exhibit Bactericidal Activity Against the M. abscessus Complex}

Time kill assays were performed using TZD for one isolate each of $M$. abscessus $(\mathrm{MIC}=2 \mathrm{mg} / \mathrm{L})$, M. bolletii $(\mathrm{MIC}=$ $8 \mathrm{mg} / \mathrm{L}$ ) and $M$. massiliense ( $\mathrm{MIC}=0.25 \mathrm{mg} / \mathrm{L})$. TZD did not exhibit bactericidal activity in all three subspecies, even at concentrations of 4- and 8-fold higher than the MIC determined by the microdilution method (Figure 1). There was a general decline in CFU count over time for M. bolletii and M. massiliense. Bacterial regrowth $\left(0.2 \log _{10} \mathrm{CFU} / \mathrm{ml}\right.$ greater than the starting inoculum) was observed at time point $72 \mathrm{~h}$ for $M$. abscessus for all drug concentrations $(0.5-8 \mathrm{x}$ MIC), following which a reduction in CFU was only observed at concentrations of $4 \mathrm{x}$ and $8 \mathrm{x}$ MIC. For M. bolletii, regrowth was noted at time point $120 \mathrm{~h}$ for concentrations of $0.25 \mathrm{x}$ and $1 \mathrm{x}$ MIC. Regrowth was observed at time point $12 \mathrm{~h}$ for $0.25 \mathrm{x}$ MIC and $72 \mathrm{~h}$ for $0.5 \mathrm{x}$ MIC for $M$. massiliense (Figure 1). 

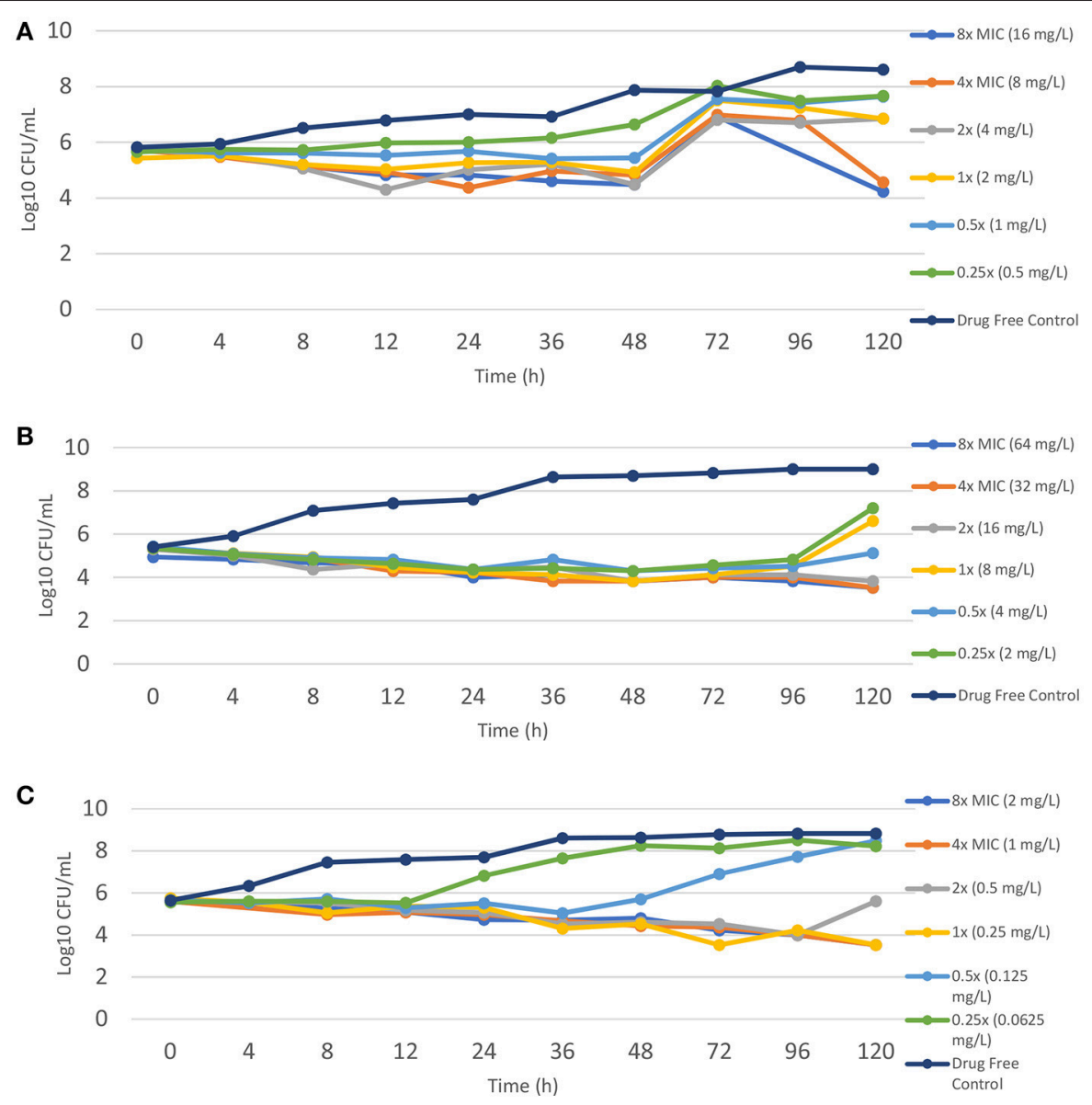

FIGURE 1 | Time kill kinetics of tedizolid against different M. abscessus subspecies (A) M. abscessus (TZD MIC = 2 mg/L); (B) M. bolletii (TZD MIC = 8 mg/L); M. massiliense (TZD MIC $=0.5 \mathrm{mg} / \mathrm{L})$. Each point represents the mean of duplicate determinations.

\section{TZD Exhibits Bacteriostatic Activity Against $M$. abscessus Complex}

TZD exhibited bacteriostatic activity against all tested isolates of the M. abscessus complex (Table 2). The MBC of all three subspecies was greater than four times of MIC, which is characteristic of a bacteriostatic antimicrobial agent.

\section{TZD Pre-exposure Does Not Induce Resistance}

Pre-treatment of three M. abscessus, M. bolletii and M. massiliense isolates to sub-inhibitory concentrations of TZD did not affect MICs (Table 3). MICs after pre-exposure to TZD were similar to those without pre-exposure, suggesting that M. abscessus did not harbor inducible TZD resistance mechanisms.

\section{Checkerboard Testing of TZD in Combination With Clarithromycin, Doxycycline and Amikacin Suggests Interactions That Are Largely Indifferent}

Amikacin (AMK) and clarithromycin (CLR) are currently the only two antimicrobial agents with reliable in vitro activity against $M$. abscessus (Tang et al., 2015). TZD in combination with CLR, DOX and AMK were evaluated for antimicrobial activity against five isolates of $M$. abscessus, four isolates of M. bolletii and five isolates of $M$. massiliense by checkerboard synergy approach (Table 4). No instances of antagonism were observed in any antimicrobial combination tested. Indifference was the primary interaction accounting for $90.5 \%$ of all interactions. For the combination of TZD and CLR, all interactions were indifferent. One instance of synergistic interaction was observed in the erm(41) C28 sequevar $M$. bolletii isolate for the combination of TZD and DOX. In two erm(41) T28 sequevar M. abscessus isolates and one $M$. massiliense isolate, synergistic interactions were observed for the combination of TZD and AMK (Table 4). Overall, the findings suggest that TZD has no interaction when used in combination with CLR, DOX and AMK against $M$. abscessus.

\section{DISCUSSION}

M. abscessus pulmonary infections are notoriously difficult to treat with low cure rates of $30-50 \%$ (5). This has 
TABLE 2 | Determination of the MBC and antibacterial mode of tedizolid against the Mycobacterium abscessus complex.

\begin{tabular}{|c|c|c|c|c|c|}
\hline $\begin{array}{l}\text { M. abscessus } \\
\text { complex }\end{array}$ & $\begin{array}{c}\text { MBC range } \\
\text { (mg/L) }\end{array}$ & $\begin{array}{c}\text { Median MBC } \\
(\mathrm{mg} / \mathrm{L})\end{array}$ & $\begin{array}{c}\text { MIC range } \\
\text { (mg/L) }\end{array}$ & $\begin{array}{c}\text { Median MIC } \\
(\mathrm{mg} / \mathrm{L})\end{array}$ & Mode of action \\
\hline $\begin{array}{l}\text { M. abscessus } \\
(n=7)\end{array}$ & $>64$ & $>64$ & $0.125-8$ & 4 & Bacteriostatic \\
\hline $\begin{array}{l}\text { M. bolletii } \\
(n=5)\end{array}$ & $8->64$ & 32 & $1-8$ & 4 & Bacteriostatic \\
\hline $\begin{array}{l}\text { M. massiliense } \\
(n=15)\end{array}$ & $16->64$ & $>64$ & $0.125-8$ & 0.5 & Bacteriostatic \\
\hline
\end{tabular}

TABLE 3 | MICs of Mycobacterium abscessus complex after exposure to sub-inhibitory concentrations of tedizolid.

\begin{tabular}{|c|c|c|}
\hline \multirow[t]{2}{*}{ M. abscessus complex* } & \multicolumn{2}{|c|}{ TZD MIC (mg/L) } \\
\hline & No pre-exposure & After pre-exposure \\
\hline M. abscessus \#1 & 4 & 2 \\
\hline M. abscessus \#2 & 4 & 2 \\
\hline M. abscessus \#3 & 8 & 4 \\
\hline M. bolletii \#1 & 0.5 & 1 \\
\hline M. bolletii \#2 & 0.5 & 0.5 \\
\hline M. bolletii \#3 & 4 & 2 \\
\hline M. massiliense \#1 & 4 & 2 \\
\hline M. massiliense \#2 & 4 & 4 \\
\hline M. massiliense \#3 & 4 & 4 \\
\hline
\end{tabular}

"Three unique isolates of each subspecies were used for testing.

spurred drug repurposing, defined as the "off-label" usage of existing antimicrobials (Palomino and Martin, 2014). LZD was initially developed for the treatment of infections caused by $\beta$-lactam-resistant Gram-positive bacteria, but it is now a recommended second-line drug for the treatment of MDR and extensively drug-resistant tuberculosis (Dheda et al., 2017). Furthermore, TZD has demonstrated in vitro activity against mycobacterial pathogens such as Mycobacterium tuberculosis and Mycobacterium fortuitum (Kisgen et al., 2014).

In our study, the potential of TZD for the treatment of $M$. abscessus complex infections was investigated in vitro. In comparison to two recent studies, our TZD MIC range of $0.0625-$ $8 \mathrm{mg} / \mathrm{L}\left(\mathrm{MIC}_{50}=1 \mathrm{mg} / \mathrm{L}, \mathrm{MIC}_{90}=4 \mathrm{mg} / \mathrm{L}\right)$ for $43 \mathrm{M}$. abscessus isolates was lower than the MIC range of $0.12->32 \mu \mathrm{g} / \mathrm{mL}$ $\left(\mathrm{MIC}_{50}=4 \mu \mathrm{g} / \mathrm{mL}, \mathrm{MIC}_{90}=8 \mu \mathrm{g} / \mathrm{mL}\right)$ reported by BrownElliott and Wallace (2017) and the MIC range of $1-16 \mu \mathrm{g} / \mathrm{mL}$ $\left(\mathrm{MIC}_{50}=2 \mu \mathrm{g} / \mathrm{mL}, \mathrm{MIC}_{90}=8 \mu \mathrm{g} / \mathrm{mL}\right)$ reported by Compain et al. (2018). For the $82 \mathrm{M}$. massiliense isolates a TZD MIC range of $0.0625-8 \mathrm{mg} / \mathrm{L}\left(\mathrm{MIC}_{50}=1 \mathrm{mg} / \mathrm{mL}, \mathrm{MIC}_{90}=4 \mathrm{mg} / \mathrm{mL}\right)$ was obtained. Brown-Elliott \& Wallace Jr. reported a TZD MIC range of $0.12->32 \mu \mathrm{g} / \mathrm{mL}\left(\mathrm{MIC}_{50}=2 \mu \mathrm{g} / \mathrm{mL}, \mathrm{MIC}_{90}=4 \mu \mathrm{g} / \mathrm{mL}\right)$ for a smaller set of 12 isolates whilst Compain et al. reported a MIC range of $1-8 \mu \mathrm{g} / \mathrm{mL}\left(\mathrm{MIC}_{50}=4 \mu \mathrm{g} / \mathrm{mL}, \mathrm{MIC}_{90}=8 \mu \mathrm{g} / \mathrm{mL}\right)$ for $14 M$. massiliense isolates $(\mathrm{n}=14)$. The TZD MIC range of $1-8$ $\mathrm{mg} / \mathrm{L}\left(\mathrm{MIC}_{50}\right.$ and $\left.\mathrm{MIC}_{90}=4 \mathrm{mg} / \mathrm{L}\right)$ for $5 \mathrm{M}$. bolletii isolates were comparable to the MIC range of $1-4 \mathrm{mg} / \mathrm{L}\left(\mathrm{MIC}_{50}=2 \mu \mathrm{g} / \mathrm{mL}\right.$, $\mathrm{MIC}_{90}=4 \mu \mathrm{g} / \mathrm{mL}$ ) as determined by Compain et al. (2018).
There are currently no CLSI recommended TZD breakpoints for Mycobacteria but LZD is considered a reliable surrogate antimicrobial agent for TZD susceptibility, with the European Committee on Antimicrobial Susceptibility Testing (EUCAST) recommending the reporting of isolates susceptible to LZD as also susceptible to TZD (EUCAST, 2016). LZD susceptibility was found to be highly predictive of TZD susceptibility, with high categorical agreement between MIC values of LZD and TZD, and low rates of very major errors for Gram-positive bacteria (e.g., Staphylococcus spp. and Enterococcus spp.) (Zurenko et al., 2014). All 130 M. abscessus isolates were susceptible to TZD when a breakpoint $\leq 8 \mathrm{mg} / \mathrm{L}$ was applied. Although the suitability of LZD as a surrogate for TZD susceptibility has only been recommended for Gram-positive bacteria, these findings suggest that M. abscessus may be more susceptible to TZD than LZD.

In $M$. tuberculosis oxazolidinone resistance is associated with point mutations in the $23 \mathrm{~S}$ rRNA gene $(\mathrm{rrl})$ and in the 50S ribosomal protein L3 (Klitgaard et al., 2015; McNeil et al., 2017). rrl and L3 mutant strains were resistant to LZD and crossresistant to sutezolid, a next-generation oxazolidinone currently in clinical development with improved potency against $M$. tuberculosis (McNeil et al., 2017). Furthermore, Gram-positive bacteria with $23 \mathrm{~S}$ rRNA gene mutations were found to have high LZD $(16 \mathrm{mg} / \mathrm{L})$ and TZD MICs $(>1 \mathrm{mg} / \mathrm{L})$ as reported in a 2011-2012 surveillance report of TZD activity (Bensaci and Sahm, 2017). In our study, 5 isolates with high TZD MIC (8 $\mathrm{mg} / \mathrm{L}$ ) had their full-length $r r l$ gene sequenced. All the sequenced isolates possessed a wild type $\mathrm{rrl}$ gene. This suggests alternative resistance mechanisms, such as efflux pumps (Gupta et al., 2006). We acknowledge our study limitation where mutations in bacterial $50 \mathrm{~S}$ ribosomal protein $\mathrm{L} 3$, which are associated with oxazolidinone resistance, were not investigated.

This is currently the first study (to the best of our knowledge) to perform a time-kill assay for all three subspecies of the $M$. abscessus complex. TZD exhibits little concentration-dependent killing and no significant bactericidal activity against the three subspecies at all tested drug concentrations $(0.5 \mathrm{x}-8 \mathrm{x}$ MIC). Compain et al. reported similar time-kill kinetics for $M$. abscessus ATCC 19977/CIP 104536, with no bactericidal activity at TZD concentrations of 4 and $8 \mathrm{mg} / \mathrm{L}$. Bacterial regrowth was observed in $M$. abscessus in the logarithmic phase of growth for TZD concentrations tested. In comparison, regrowth was only observed at lower TZD concentrations of $0.25 \mathrm{x}$ and $1 \mathrm{x}$ MIC for $M$. bolletii and $0.25 \mathrm{x}$ and $0.5 \mathrm{x}$ MIC for M. massiliense. These findings were consistent with the findings by Ferro et al. (2015) 
TABLE 4 | FIC index for tedizolid tested in combination with clarithromycin, doxycycline and amikacin against the Mycobacterium abscessus complex.

\begin{tabular}{|c|c|c|c|c|c|c|c|c|c|c|c|c|}
\hline \multirow[b]{2}{*}{$\begin{array}{l}\text { M. abscessus } \\
\text { complex }\end{array}$} & \multirow[b]{2}{*}{$\begin{array}{l}\text { Susceptibility } \\
\text { to CLR }\end{array}$} & \multirow[b]{2}{*}{ erm $(41)^{\$}$} & \multicolumn{4}{|c|}{ MIC (mg/L) } & \multirow{2}{*}{$\begin{array}{l}\text { FIC index } \# \\
\text { TZD + CLR }\end{array}$} & \multirow{2}{*}{ Interaction } & \multirow{2}{*}{$\begin{array}{l}\text { FIC index } \# \\
\text { TZD + DOX }\end{array}$} & \multirow{2}{*}{ Interaction } & \multirow{2}{*}{$\begin{array}{c}\text { FIC index } \# \\
\text { TZD + AMK }\end{array}$} & \multirow{2}{*}{ Interaction } \\
\hline & & & TZD & CLR & DOX & AMK & & & & & & \\
\hline $\begin{array}{l}\text { M. abscessus } \\
(n=1)\end{array}$ & $S$ & C28 sequevar & 1 & 2 & 1 & 1 & 1.000 & Indifference & 1.000 & Indifference & 0.625 & Indifference \\
\hline $\begin{array}{l}\text { M. abscessus } \\
(n=4)\end{array}$ & $\mathrm{R}$ & T28 sequevar & 3 & $>16$ & 1 & 1 & 1.000 & Indifference & 1.000 & Indifference & 0.625 & Indifference \\
\hline M. abscessus & $\mathrm{R}$ & T28 sequevar & 4 & $>16$ & 1 & 16 & 1.016 & Indifference & 0.625 & Indifference & 0.375 & Synergistic \\
\hline M. abscessus & $\mathrm{R}$ & T28 sequevar & 1 & $>16$ & 1 & 2 & 1.016 & Indifference & 1.000 & Indifference & 1.063 & Indifference \\
\hline M. abscessus & $\mathrm{R}$ & T28 sequevar & 8 & $>16$ & 1 & 2 & 1.016 & Indifference & 1.125 & Indifference & 0.750 & Indifference \\
\hline M. bolletii $(n=1)$ & S & C28 sequevar & 1 & 1 & 8 & 16 & 1.016 & Indifference & 0.313 & Synergistic & 0.53 & Indifference \\
\hline M. bolletii $(n=3)$ & $\mathrm{R}$ & T28 sequevar & 8 & $>16$ & 2 & 16 & 1.016 & Indifference & 0.563 & Indifference & 1.008 & Indifference \\
\hline M. bolletii & $\mathrm{R}$ & T28 sequevar & 4 & $>16$ & 1 & 8 & 1.500 & Indifference & 0.625 & Indifference & 0.563 & Indifference \\
\hline M. bolletii & $\mathrm{R}$ & T28 sequevar & 4 & $>16$ & 4 & 0.5 & 1.016 & Indifference & 0.625 & Indifference & 0.750 & Indifference \\
\hline $\begin{array}{l}\text { M. massiliense } \\
(n=5)\end{array}$ & S & Deleted & 0.25 & 0.25 & 2 & 2 & 1.016 & Indifference & 0.563 & Indifference & 0.625 & Indifference \\
\hline M. massiliense & S & Deleted & 2 & 2 & 1 & 1 & 2.016 & Indifference & 1.000 & Indifference & 0.750 & Indifference \\
\hline M. massiliense & S & Deleted & 1 & 1 & 2 & 2 & 0.516 & Indifference & 0.625 & Indifference & 0.375 & Synergistic \\
\hline M. massiliense & S & Deleted & 0.125 & 0.125 & 1 & 2 & 1.016 & Indifference & 0.750 & Indifference & 0.750 & Indifference \\
\hline M. massiliense & $S$ & Deleted & 1 & 1 & 1 & 1 & 1.016 & Indifference & 0.750 & Indifference & 1.000 & Indifference \\
\hline
\end{tabular}

\$T28 sequevar are CLR resistant, C28 sequevars are CLR susceptible. Deleted, refers to 274 bp erm(41) gene deletion characteristic in the M. massiliense subspecies. \#FIC index was calculated as [(MIC of tedizolid in combination/MIC of tedizolid alone) + (MIC of second antibiotic in combination/MIC of second antibiotic alone)].

Only FIC index $<0.5$ was considered as a synergistic interaction.

where regrowth was also observed for AMK and CLR after 72 hours even at concentrations of $2 \mathrm{x}$ to $8 \mathrm{x}$ MIC. The findings suggest that a TZD concentration of $\geq 4 \mathrm{x}$ MIC may be required to induce significant killing activity against $M$. abscessus, whereas a TZD concentration of $1 \mathrm{x} / 2 \mathrm{x}$ MIC sufficiently reduces bacterial counts in M. bolletii and M. massiliense over time. Similar to other active antimicrobials against $M$. abscessus complex, TZD exhibits a bacteriostatic effect that is more pronounced in $M$. bolletii and M. massiliense than M. abscessus.

Synergy studies of TZD with CLR, DOX, AMK demonstrated that all combinations primarily showed indifferent interactions with no instances of antagonism. A similar study performed by Compain et al. reported indifferent interactions of TZD

\section{REFERENCES}

Aziz, D. B., Low, J. L., Wu, M. L., Gengenbacher, M., Teo, J. W. P., Dartois, V., et al. (2017). Rifabutin Is active against Mycobacterium abscessus complex. Antimicrob. Agents Chemother. 61:e00155-e00117.

Bensaci, M., and Sahm, D. (2017). Surveillance of tedizolid activity and resistance: in vitro susceptibility of Gram-positive pathogens collected over 5 years from the United States and Europe. Diagn. Microbiol. Infect. Dis. 87, 133-138. doi: 10.1016/j.diagmicrobio.2016. 10.009

Benwill, J. L., and Wallace, R. J. (2014). Mycobacterium abscessus: challenges in diagnosis and treatment. Curr. Opin. Infect. Dis. 27, 506-510. doi: $10.1097 / Q C O .0000000000000104$

Brown-Elliott, B. A., and Wallace, R. J. (2017). In Vitro Susceptibility testing of tedizolid against nontuberculous Mycobacteria. J. Clin. Microbiol. 55, 1747-1754. doi: 10.1128/JCM.00274-17 with TGC, AMK and ciprofloxacin, with the combination of CLR and TZD showing one synergistic interaction out of 6 tested isolates. The findings suggest that TZD could be used in the existing combination regime of CLR and AMK with no antagnostic interactions.

\section{AUTHOR CONTRIBUTIONS}

YT drafted the manuscript and performed the experiments. BC performed the experiments. SY provided clinical feedback and scientific review. RL provided clinical feedback and scientific review. JT drafted the manuscript and oversaw the project execution.

Chew, K. L., Cheng, J. W. S., Hudaa Osman, N., Lin, R. T. P., and Teo, J. W. P. (2017). Predominance of clarithromycin-susceptible Mycobacterium massiliense subspecies: characterization of the Mycobacterium abscessus complex at a tertiary acute care hospital. J. Med. Microbiol. 66, 1443-1447. doi: $10.1099 / \mathrm{jmm} \cdot 0.000576$

Choi, G. E., Shin, S. J., Won, C. J., Min, K. N., Oh, T., Hahn, M. Y., et al. (2012). Macrolide treatment for mycobacterium abscessus and Mycobacterium massiliense infection and inducible resistance. Am. J. Respir. Crit. Care Med. 18, 917-925. doi: 10.1164/rccm.201111-2005OC

CLSI (1999). Methods for determining bactericidal activity of antimicrobial agents; approved guideline M26-A. Clin. Lab. Stand. Inst. 19:7.

CLSI (2015). Methods for Dilution Antimicrobial Susceptibility Tests for Bacteria That Grow Aerobically. Approved Standard, 10th Edn. CLSI document M07A10, 1-87.

CLSI (2017). Performance Standards for Antimicrobial Susceptibility Testing. Perform Stand Antimicrob susceptibility Test 282. 
Compain, F., Soroka, D., Heym, B., Gaillard, J. L., Herrmann, J. L., Dorchène, D., et al. (2018). In vitro activity of tedizolid against the Mycobacterium abscessus complex. Diagn. Microbiol. Infect. Dis. 90, 186-189. doi: 10.1016/j.diagmicrobio.2017.11.001

Dheda, K., Gumbo, T., Maartens, G., Dooley, K. E., McNerney, R., Murray, M., et al. (2017). The epidemiology, pathogenesis, transmission, diagnosis, and management of multidrug-resistant, extensively drug-resistant, and incurable tuberculosis. Lancet Respir. Med. doi: 10.1016/S2213-2600(17)30079-6. [Epub ahead of print].

EUCAST (2016). Breakpoint Tables for Interpretation of MICs and Zone Diameters European Committee on Antimicrobial Susceptibility Testing Breakpoint Tables for Interpretation of MICs and Zone Diameters. Växjö: EUCAST Development Laboratory for Antimicrobial Susceptibility Testing of bacteria c/o Clinical Microbiology.

Ferro, B. E., Van Ingen, J., Wattenberg, M., Van Soolingen, D., and Mouton, J. W. (2015). Time-kill kinetics of antibiotics active against rapidly growing mycobacteria. J. Antimicrob. Chemother. 70, 811-817. doi: 10.1093/jac/dku431

Gupta, A. K., Chauhan, D. S., Srivastava, K., Das, R., Batra, S., Mittal, M., et al. (2006). Estimation of efflux mediated multi-drug resistance and its correlation with expression levels of two major efflux pumps in Mycobacteria. J. Commun. Dis. 38, 246-254.

Huang, Y.C., Liu, M.F., Shen, G.H., Lin, C.F., Kao, C.C., Liu, P.Y., et al. (2010). Clinical outcome of Mycobacterium abscessus infection and antimicrobial susceptibility testing. J. Microbiol. Immunol. Infect. 43, 401-406. doi: 10.1016/S1684-1182(10)60063-1

Kaushik, A., Makkar, N., Pandey, P., Parrish, N., Singh, U., and Lamichhane, G. (2015). Carbapenems and rifampin exhibit synergy against Mycobacterium tuberculosis and Mycobacterium abscessus. Antimicrob. Agents Chemother. 59, 6561-6567. doi: 10.1128/AAC.01158-15

Kisgen, J. J., Mansour, H., Unger, N. R., and Childs, L. M. (2014). Tedizolid: A new oxazolidinone antimicrobial. Am. J. Health Syst. Pharm. 71, 621-633. doi: 10.2146/ajhp130482

Klitgaard, R. N., Ntokou, E., Nørgaard, K., Biltoft, D., Hansen, L. H., Trædholm, N. M., et al. (2015). Mutations in the bacterial ribosomal protein 13 and their association with antibiotic resistance. Antimicrob. Agents Chemother. 59, 3518-3528. doi: 10.1128/AAC.00179-15

Koh, W. J., Jeon, K., Lee, N. Y., Kim, B. J., Kook, Y. H., Lee, S. H., et al. (2011). Clinical significance of differentiation of Mycobacterium massiliense from Mycobacterium abscessus. Am. J. Respir. Crit. Care Med. 183, 405-410. doi: 10.1164/rccm.201003-0395OC

Lee, M. R., Sheng, W. H., Hung, C. C., Yu, C. J., Lee, L. N., and Hsueh, P. R. (2015). Mycobacterium abscessus complex infections in humans. Emerging Infect. Dis. 21, 1638-1646. doi: 10.3201/2109.141634

Macheras, E., Roux, A. L., Bastian, S., Leão, S. C., Palaci, M., Sivadon-Tardy, V., et al. (2011). Multilocus sequence analysis and rpoB sequencing of Mycobacterium abscessus (sensu lato) strains. J. Clin. Microbiol. 49, 491-499. doi: 10.1128/JCM.01274-10

McNeil, M. B., Dennison, D. D., Shelton, C., and Parish, T. (2017). In vitro isolation and characterization of oxazolidinone resistant Mycobacterium tuberculosis.
Antimicrob. Agents Chemother. 61:e01296-e01217. doi: 10.1128/AAC. 01296-17

Moran, G. J., Fang, E., Corey, G. R., Das, A. F., De Anda, C., and Prokocimer, P. (2014). Tedizolid for 6 days versus linezolid for 10 days for acute bacterial skin and skin-structure infections (ESTABLISH-2): A randomised, double-blind, phase 3, non-inferiority trial. Lancet Infect. Dis. 14, 696-705. doi: 10.1016/S1473-3099(14)70737-6

Nessar, R., Cambau, E., Reyrat, J. M., Murray, A., and Gicquel, B. (2012). Mycobacterium abscessus: A new antibiotic nightmare. J. Antimicrob. Chemother. 67, 810-818. doi: 10.1093/jac/dkr578

Palomino, J. C., and Martin, A. (2014). Drug resistance mechanisms in Mycobacterium tuberculosis. Antibiotics (Basel). 2, 317-340. doi: 10.3390/antibiotics3030317

Rubio, M., March, F., Garrigó, M., Moreno, C., Español, M., and Coll, P. (2015). Inducible and acquired clarithromycin resistance in the Mycobacterium abscessus complex. PLoS ONE 10:e0140166. doi: 10.1371/journal.pone.0140166

Rybak, J. M., Marx, K., and Martin, C. A. (2014). Early experience with tedizolid: clinical efficacy, pharmacodynamics, and resistance. Pharmacotherapy. 34, 1198-1208. doi: 10.1002/phar.1491

Tang, S. S., Lye, D. C., Jureen, R., Sng, L. H., and Hsu, L. Y. (2015). Rapidly growing mycobacteria in Singapore, 2006-2011. Clin. Microbiol. Infect. 21, 236-241. doi: 10.1016/j.cmi.2014.10.018

Van Ingen, J., Boeree, M. J., Van Soolingen, D., and Mouton, J. W. (2012). Resistance mechanisms and drug susceptibility testing of nontuberculous mycobacteria. Drug Resistance Updates 15, 149-161. doi: 10.1016/j.drup.2012.04.001

Woods, G. L., Brown-Elliott, B. A., Conville, P. S., Desmond, E. P., Hall, G. S., and Lin, G. (2011). Susceptibility testing of mycobacteria, nocardiae, and other aerobic actinomycetes; approved standard-second edition. Clin. Lab. Stand. Inst. 26, 1-61.

Zurenko, G., Bien, P., Bensaci, M., Patel, H. N., and Thorne, G. (2014). Use of linezolid susceptibility test results as a surrogate for the susceptibility of grampositive pathogens to tedizolid, a novel oxazolidinone. Ann. Clin. Microbiol. Antimicrob. 13:46. doi: 10.1186/s12941-014-0046-0

Conflict of Interest Statement: The authors declare that the research was conducted in the absence of any commercial or financial relationships that could be construed as a potential conflict of interest.

The handling editor declared a past co-authorship with one of the authors JT.

Copyright (c) 2018 Tang, Cheng, Yeoh, Lin and Teo. This is an open-access article distributed under the terms of the Creative Commons Attribution License (CC BY). The use, distribution or reproduction in other forums is permitted, provided the original author(s) and the copyright owner(s) are credited and that the original publication in this journal is cited, in accordance with accepted academic practice. No use, distribution or reproduction is permitted which does not comply with these terms. 